Elsevier required licence: (C) $<2015>$. This manuscript version is made available under the CC-BY-NC-ND 4.0 license http://creativecommons.org/licenses/by-nc-nd/4.0/ 


\title{
Nano-Sensing of the Orientation of Fluorescing Molecules with Active Coated Nano-Particles
}

\author{
Samel Arslanagić, and Richard W. Ziolkowski
}

\begin{abstract}
The potential of using active coated nano-particles to determine the orientation of fluorescing molecules is reported. By treating each fluorescing molecule as an electric Hertzian dipole, single and multiple fluorescing molecules emitting coherently and incoherently in various orientations are considered in the presence of active coated nano-particles. It is demonstrated that in addition to offering a means to determine the orientation of a single molecule or the over-all orientation of the molecules surrounding it, the nature of the far-field response from the active coated nano-particles provides insight into the degree of phase coherence between emitters in specific molecular environments.
\end{abstract}

Keywords Terms-Gain, nano-particles, molecules, plasmons, resonances, light radiation and scattering

\section{INTRODUCTION}

$\mathrm{F}$ luorescence spectroscopy techniques have become a mainstay in many fields associated with cellular and molecular imaging [1]. They can be used to determine molecular properties down to single-molecule levels [2-6]. The measurements of several single-molecule photo-physical parameters (e.g., distances, emission rates, lifetimes, quantum yields, resonant energy transfer) depend critically on the orientation of the molecule's absorption- or emission-dipole moment [7-10].

As suggested in the seminal paper by Purcell [11], it is now well known that the lifetime of an excited atomic state is impacted by its local environment. There have been a variety of investigations into how this effect impacts the properties of fluorescence. It was demonstrated that the fluorescence process near an interface can be modeled as the emission of an electric Hertzian dipole (EHD) oriented along the molecule's dipole transition moment in the same local environment [12]. This approach was followed to understand how a single molecule's orientation can be determined from its emission pattern in the presence of glass coverslip. The resulting measurement techniques rely on the presence of a planar substrate on which the effective dipole is located and optical systems to record the emissions [10]. More localized approaches have been sought that are more nano-technology based. For instance, the Purcell-fluoresence connection was further explored in experimental and theoretical studies of the

S. Arslanagic is with the Department of Electrical Engineering, Technical University of Denmark, DK-2800 Kgs. Lyngby, Denmark ( phone: +45 4525 3825; fax: +4593 1634; e-mail: sar@elektro.dtu.dk).

R. W. Ziolkowski is with the Department of Electrical and Computer Engineering Department, University of Arizona, 1230 E. Speedway Blvd., Tucson, 85721-0104, USA (e-mail: ziolkows@email.arizona.edu). fluorescence rate of a single molecule as a function of its distance from a laser-irradiated gold nanoparticle [13] and in terms of the gold nano-particle acting as an optical nanoantenna [14]. The introduction of other types of optical nanoantennas near fluorescing molecules also has demonstrated the ability to control their emissions [15].

Contrary to these previous works, that rely either on the presence of cumbersome substrates or passive nano-particles, the present work considers the use of coated (core-shell) nanoparticles in which gain is encapsulated in their core that act as optical nano-antennas for effective determination of the orientation of fluorescing molecules. It is shown that highly sub-wavelength active nano-particles provide extremely sensitive far-field signatures that highly depend on the orientation, as well as the degree of phase coherence of the surrounding sensed molecules, thereby rendering their use for very effective determination of the orientation of a singlemolecule and multiple molecules undergoing fluorescence. The manuscript is organized as follows. In Section II the configuration of a CNP excited by one, two, or four EHDs is described. Section III includes the necessary details of the analytical treatment. Section IV is initiated by a brief account on the material and gain models used in the active CNP

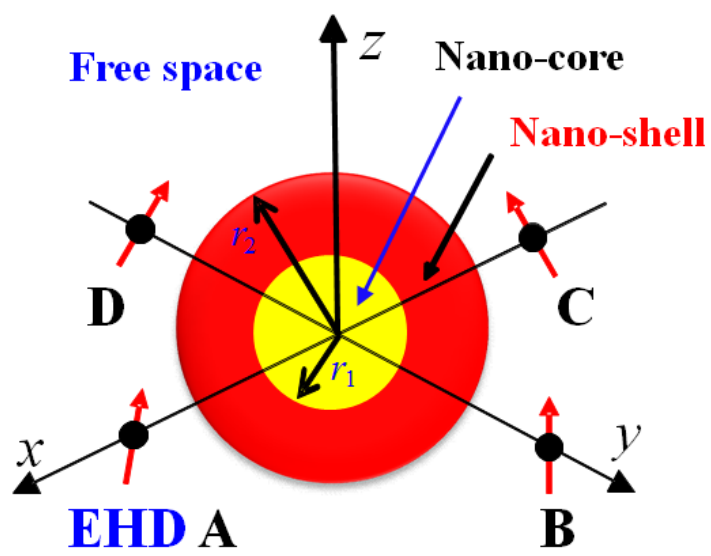

Fig.1. Multiple electric Hertzian dipoles (EHDs) illuminate a spherical CNP. A gain impregnated $\mathrm{SiO}_{2}$ core is coated with a Ag shell. The EHDs are represented by the black circles. Three excitation cases are considered: Case 1 (EHD A), Case 2 (EHD $\mathrm{A}+\mathrm{C})$ and Case $3(\mathrm{EHD} \mathrm{A}+\mathrm{B}+\mathrm{C}+\mathrm{D})$. Within each of the excitation cases, the EHDs oriented tangentially or normally, as well as their combinations, with respect to the CNP surface are investigated. 
configuration. This discussion is followed by a presentation of the highlighted numerical results. Finally, Section V summarizes and concludes the present work, and outlines ideas for its extensions. Throughout the manuscript, the time factor $\exp (j \omega t)$, with $\omega$ being the angular frequency, and $t$ being the time, is assumed and suppressed.

\section{CONFIGURATION}

The spherical CNP configuration that will be used to sense the molecular orientation is shown in Figure 1. It consists of a nano-core (radius $r_{1}$ ) covered concentrically with a spherical nano-shell (outer radius $r_{2}$ ), and is immersed in free space. The nano-core and nano-shell have the permittivities, permeabilities, and wave numbers, respectively, denoted by $\varepsilon_{1}, \mu_{1}$, and $k_{1}$, (for the nano-core) and $\varepsilon_{2}, \mu_{2}$, and $k_{2}$ (for the nanoshell). The permittivity and permeability of the ambient free space are $\varepsilon_{0}$ and $\mu_{0}$; the corresponding wave number is $k_{0}=\omega \sqrt{\varepsilon_{0} \mu_{0}}$, and the intrinsic impedance is $\eta_{0}=\sqrt{\mu_{0} / \varepsilon_{0}}$.

The CNP in Figure 1 is excited by one or more electric Hertzian dipoles (EHDs), which, following [12], constitute our model of the surrounding molecules. These EHDs are represented by the red arrows overlayed with the black circles and are labeled as A, B, C, and D, in the figure. This is to imply that they can be arbitrarily oriented with the dipole moment of the Xth EHD, $\mathrm{X}=\mathrm{A}, \mathrm{B}, \mathrm{C}$, and $\mathrm{D}$, being equal to $\vec{p}_{\mathrm{X}}=\hat{p}_{\mathrm{X}} p_{\mathrm{X}}$, where $\hat{p}_{\mathrm{X}}$ is its orientation and $p_{\mathrm{X}}[\mathrm{Am}]$ is its complex amplitude. A spherical coordinate system $(r, \theta, \phi)$ and the associated rectangular coordinate system $(x, y, z)$ are introduced such that their origins coincide with the center of the CNP.

Three specific excitation cases of the CNP in Figure 1 are investigated: Case 1, where only EHD A excites the CNP; Case 2, where EHD A and C excite the CNP; and Case 3, where all four dipoles excite the CNP simultaneously. The exciting EHDs are located symmetrically at positions in the $x y$-plane outside the CNP, i.e., EHDs A and C are centered on opposite sides of the CNP along the $x$-axis, whereas EHDs B and $\mathrm{D}$ are centered on opposite sides along the $y$-axis. The coordinates of the observation point are $(r, \theta, \phi)$, and those of the EHDs are $\left(r_{s}, \theta_{s}=\pi / 2\right)$, where $\phi_{s}=0$ (EHD A), $\pi / 2$ (EHD B), $\pi$ (EHD C) and $3 \pi / 2$ (EHD D).

Within each of the excitation cases, both tangential and normal EHD orientations with respect to the CNP surface, as well as combination of these, are examined. In particular, we consider in detail:

Case $1(\mathrm{X}=\mathrm{A})$ :

a) $\hat{p}_{\mathrm{A}}=\hat{z} ;$ b) $\hat{p}_{\mathrm{A}}=\hat{x}$

Case $2(\mathrm{X}=\mathrm{A}$ and $\mathrm{C})$ :
a) $\hat{p}_{\mathrm{X}}=\hat{z}$;
b) $\hat{p}_{\mathrm{A}}=\hat{z}, \quad \hat{p}_{\mathrm{C}}=\hat{x}$;
c) $\hat{p}_{\mathrm{X}}=\hat{x}$

Case $3(\mathrm{X}=\mathrm{A}, \mathrm{B}, \mathrm{C}$, and $\mathrm{D})$ :
a) $\hat{p}_{\mathrm{X}}=\hat{z}$;
b) $\hat{p}_{\mathrm{A}, \mathrm{C}}=\hat{x}, \hat{p}_{\mathrm{B}, \mathrm{D}}=\hat{z}$;
c) $\hat{p}_{\mathrm{A}, \mathrm{C}}=\hat{x}, \hat{p}_{\mathrm{B}, \mathrm{D}}=\hat{y}$.

If a given case, Case $i, \mathrm{i}=1,2$, and 3 , considers EHDs with only tangential (normal) orientations, it will be referred to as Case $i / \mathrm{T}(\mathrm{N})$. On the other hand, it will be referred to as Case $\mathrm{i} / \mathrm{TN}$ if its sources have both of these orientations.

\section{THEORY}

The analytical solution related to the Case 1 excitation in Figure 1 was derived in [16], and was later on extended in [17] to deal with the other two excitation cases. Below, we report only the pertinent details of these solutions.

The electromagnetic field due to any of the EHDs ( $\mathrm{X}=\mathrm{A}, \mathrm{B}$, $C$ or D) is expanded in transverse magnetic (TM) and transverse electric (TE) spherical vector waves as

$$
\vec{E}=\sum_{n=1}^{\infty} \sum_{m=-n}^{n} \frac{1}{j \omega \varepsilon_{0} \mu_{0}} a_{n m, \mathrm{X}}^{(c)} \vec{N}_{n m}^{\mathrm{TM},(c)}-\frac{1}{\varepsilon_{0}} b_{n m, \mathrm{X}}^{(c)} \vec{M}_{n m}^{\mathrm{TE},(c)}
$$

where $\vec{N}_{n m}^{\mathrm{TM},(c)}$ and $\vec{M}_{n m}^{\mathrm{TE},(c)}$ are the spherical vector wave functions (given by equation (2) and (3) in [16]), while $a_{\mathrm{X}, n m}^{(c)}$ and $b_{\mathrm{X}, n m}^{(c)}$ are the TM and TE expansion coefficients, respectively, of the EHD field (given by equation (4) and (5) in [16]]. In (1), the index $c=1$ applies for $r<r_{s}$, while $c=4$ applies for $r>r_{s}$. Since the objective of this work is to assess the influence of the EHD/molecule orientation in the three excitation cases, we note that the explicit forms of the TM and TE expansion coefficients for the presently investigated three EHD orientations read

$$
\begin{gathered}
a_{n m, \mathrm{X}}^{(c)}=F_{n m} \frac{\mu_{0}}{r_{s}}\left\{w_{n}^{(5-c)}\left(k_{0} r_{s}\right)+k_{0} r_{s} w_{n}^{(5-c)^{\prime}}\left(k_{0} r_{s}\right)\right\} \\
\cdot(n+1-|m|) P_{n+1}^{|m|}(1) e^{-j m \phi_{s}} \\
b_{n m, \mathrm{X}}^{(c)}=G_{n m} \varepsilon_{0} w_{n}^{(5-c)}\left(k_{0} r_{s}\right) j m P_{n}^{|m|}(1) e^{-j m \phi_{s}}
\end{gathered}
$$

for the z-oriented $\operatorname{EHD}\left(\hat{p}_{\mathrm{X}}=\hat{z}\right)$, and

$$
\begin{aligned}
a_{n m, \mathrm{X}}^{(c)}= & F_{n m} \frac{\mu_{0}}{r_{s}} P_{n}^{|m|}(1) e^{-j m \phi_{s}} \\
& \cdot\left\{n(n+1) w_{n}^{(5-c)}\left(k_{0} r_{s}\right) \cos \left(\phi_{s}\right)\right. \\
& \left.+\left(w_{n}^{(5-c)}\left(k_{0} r_{s}\right)+k_{0} r_{s} w_{n}^{(5-c)^{\prime}}\left(k_{0} r_{s}\right)\right) j m \sin \left(\phi_{s}\right)\right\} \\
b_{n m, \mathrm{X}}^{(c)}= & G_{n m} \varepsilon_{0} w_{n}^{(5-c)}\left(k_{0} r_{s}\right)(n+1-|m|) P_{n+1}^{|m|}(1) e^{-j m \phi_{s}} \sin \left(\phi_{s}\right)
\end{aligned}
$$

for the $x$-oriented $\operatorname{EHD}\left(\hat{p}_{\mathrm{X}}=\hat{x}\right)$, and

$$
\begin{aligned}
a_{n m, \mathrm{X}}^{(c)}= & F_{n m} \frac{\mu_{0}}{r_{s}} P_{n}^{|m|}(1) e^{-j m \phi_{s}} \\
& \cdot\left\{n(n+1) w_{n}^{(5-c)}\left(k_{0} r_{s}\right) \sin \left(\phi_{s}\right)\right. \\
& \left.\quad-\left(w_{n}^{(5-c)}\left(k_{0} r_{s}\right)+k_{0} r_{s} w_{n}^{(5-c) '}\left(k_{0} r_{s}\right)\right) j m \cos \left(\phi_{s}\right)\right\}
\end{aligned}
$$


$b_{n m, \mathrm{X}}^{(c)}=-G_{n m} \varepsilon_{0} w_{n}^{(5-c)}\left(k_{0} r_{s}\right)(n+1-|m|) P_{n+1}^{|m|}(1) e^{-j m \phi_{s}} \cos \left(\phi_{s}\right)$

for the $y$-oriented EHD $\left(\hat{p}_{\mathrm{X}}=\hat{y}\right)$. In (1)-(4), the function $w_{n}^{(c)}(\cdot)$ is the spherical Bessel function of order $n, j_{n}(\cdot)$, for $c$ $=1\left(r<r_{s}\right)$, and it equals the spherical Hankel function of second kind and order $n$ for $c=4\left(r<r_{s}\right)$. Moreover, the function $P_{n}^{|m|}(\cdot)$ is the associated Legendre function of degree $n$ and order $m$. The quantities $F_{n m}$ and $G_{n m}$ in (2)-(4) take the form of

$$
\begin{aligned}
& F_{n m}=-j k_{0} \frac{p_{s, X}}{4 \pi} \frac{1}{\mu_{0}} \frac{2 n+1}{n(n+1)} \frac{(n-|m|) !}{(n+|m|) !} \\
& G_{n m}=\frac{k_{0}^{3}}{\omega} \frac{p_{s, X}}{4 \pi} \frac{1}{\varepsilon_{0}^{2}} \frac{2 n+1}{n(n+1)} \frac{(n-|m|) !}{(n+|m|) !} .
\end{aligned}
$$

The unknown fields inside and outside the CNP, are also expanded in terms of TM and TE spherical waves. These expansions involve the unknown TM and TE expansion coefficients denoted by $A_{i, n m}$ and $B_{i, n m}$, respectively, where $i=1$ for the fields inside the nano-core, $i=2$ and 3 for the fields inside the nano-shell, and $i=4$ for the fields outside the CNP. These expansion coefficients depend on the EHD location and orientation; they are easily obtained by enforcing the boundary conditions on the two spherical interfaces, $r=r_{1}$ and $r=r_{2}$. The total electric, $\vec{E}$, and magnetic, $\vec{H}$, fields in all regions will, in general, possess all three components.

With the fields in place, one can derive the expression for the total power radiated by the CNP when it is excited by the EHDs in Cases 1, 2, and 3. It is given by the expression

$$
P_{\mathrm{t}}=\frac{\pi}{\omega k_{0}} \sum_{n=1}^{N_{\max }} \sum_{m=-n}^{n} 2 \frac{n(n+1)}{2 n+1} \frac{(n+|m|) !}{(n-|m|) !}\left[\frac{\left|a_{n m}\right|^{2}}{\varepsilon_{0}}+\frac{\left|b_{n m}\right|^{2}}{\mu_{0}}\right] \text {, }
$$

where the coefficients

$$
d_{n m}=D_{4, n m}+\left\{\begin{array}{cc}
d_{A, n m}^{(4)} & \text { Case 1, } \\
d_{A, n m}^{(4)}+d_{C, n m}^{(4)} & \text { Case 2, } \\
d_{A, n m}^{(4)}+d_{B, n m}^{(4)}+d_{C, n m}^{(4)}+d_{D, n m}^{(4)} & \text { Case 3, }
\end{array}\right.
$$

with the symbols $d$ and $D$ being, respectively, the excitation coefficients $a$ and scattering coefficients $A$ for the TM terms, and $b$ and $B$ for the TE terms. The power radiated by the EHDs in Case 1, 2, and 3, when being alone in free space, is denoted by $P_{\mathrm{EHD}}$, and it results from (6) for $D_{4, n m}=0$.

In our investigations, the so-called normalized radiation resistance (NRR) is examined [18]. This quantity is the radiation resistance of the dipoles radiating in the presence of the CNP normalized by the radiation resistance of the dipoles radiating in free space. Expressed in $\mathrm{dB}$, the NRR reads

$\mathrm{NRR}(\mathrm{dB})=10 \cdot \log _{10}\left(\frac{P_{\mathrm{t}}}{P_{\mathrm{EHD}}}\right)$.

The NRR is equivalent to the well-known Purcell factor [11, $19,20]$ and to the radiated power ratio (RPR) introduced in the original metamaterial-based antenna studies [21-23]. We will continue use the NRR term here to provide continuity with previous investigations $[17,18]$.

In addition to examining the impact of the EHD/molecule orientation on the NRR (7), the field solutions will also be employed to supplement these studies with the power flow density behavior in the interior and the exterior of the CNP. The power flow is calculated in terms of the Poynting vector (magnitude and direction), which reads

$\vec{S}=\frac{1}{2} \operatorname{Re}\left\{\vec{E} \times \vec{H}^{*}\right\}=\hat{a}_{r} S_{r}+\hat{a}_{\theta} S_{\theta}+\hat{a}_{\phi} S_{\phi}$

where $S_{r}, S_{\theta}$, and $S_{\phi}$ are its $r-, \theta-$, and $\phi$-components. These are related to the components of the total electric and magnetic fields as shown in [17].

\section{BACKGROUND - MATERIAL AND GAIN MOdELS}

Previous works [17, 18, 24-29] have demonstrated that highly resonant CNPs can be designed when gain is incorporated with them even though their overall size is deeply sub-wavelength. The highly resonant nature of these active CNPs was found to lead to very large scattering cross sections [24-26, 28] (for plane wave excitation), as well as to enhanced radiated powers for the localized excitation sources $[17,18,27,29]$. These more recent results confirm expectations from earlier analyses [30 31]. Although the impact of the CNP material choice and its overall size on the resonant nature has been examined [27], significant attention in those works was devoted to a CNP consisting of a silica $\left(\mathrm{SiO}_{2}\right)$ nano-core (with $r_{1}=24 \mathrm{~nm}$ radius), coated with a silver (Ag) nano-shell $\left(6 \mathrm{~nm}\right.$ thick, i.e., $\left.r_{2}=30 \mathrm{~nm}\right)$. This basic configuration is referred to as the Ag-based CNP, and it will form the basis of the present investigations. The size and frequency dependencies of the silver nano-shell permittivity are accounted for as described in [24]. The tunability of these core-shell particles has been emphasized for a number of plasmonic applications [32-35]. In the model of the active CNP, the gain is introduced inside the lossless silica nano-core and is modeled by a constant frequency model according to which the silica nano-core permittivity takes the form of

$$
\varepsilon_{1}=\left(n^{2}-\kappa^{2}-2 j n \kappa\right) \varepsilon_{0}
$$

where $n=\sqrt{2.05}$ is the refractive index of the silica nano-core in the frequency region of interest, and the parameter $\kappa$ determines the nature of the nano-core and thus of the CNP. The nano-core is lossless and passive for $\kappa=0$, it is lossy and 
passive for $\kappa>0$, and it is active for $\kappa<0$. As shown previously [35], the active Ag-based CNPs lead to the socalled super-resonant state with very large associated radiated powers of the near-by EHDs (Case 1, 2, and 3), for $\kappa=-0.245$ at the excitation wavelength of $\lambda=502.1 \mathrm{~nm}$, and it moreover leads to severe reductions of the radiated power at $594.9 \mathrm{~nm}$ (Case 2) and $593.2 \mathrm{~nm}$ (Case 2 and 3). The influence of the EHD orientation within the respective excitation cases on the resulting maximum and minimum values of the radiated power will next be examined; this will reveal the potential of the obtained results to determine molecular orientations using active CNPs as highly localized nano-sensors.

\section{RESUlTS AND DisCUSSIONS}

We first report the results for the three excitation cases in which the individual EHDs each radiate in phase. Subsequently, we consider the Case 2 excitation in which the two EHDs are radiating in-coherently. This configuration may be the most representative of what might be expected in a realistic molecular environment. In all cases, the EHDs are located at their respective points in the $x y$-plane at a radial distance of $r_{s}=40 \mathrm{~nm}$.

It is interesting to note that since the normal ( $x$-oriented) EHDs in the Case 1, 2, and 3 excitations are located along the $\mathrm{x}$-axis, and the other two normal (y-oriented) EHDs in the Case 3 excitation are located along the y-axis, it follows from (3b) and (4b) that the TE expansion coefficients in these cases are zero. Consequently, the TM and TE modes are decoupled for these particular sets of EHD orientations in the various excitation cases.

\section{A. Phase-coherent EHDs (molecules) - Case 1, 2, and 3}

The magnitudes of the dipole moments of all EHDs are assumed identical and equal to $p_{s}=5 \mathrm{nA}-\mathrm{m}$. Figure 2 shows the NRR (dB) as a function of the excitation wavelength for the Case 1 (a), Case 2 (b), and Case 3 (c) excitations, including the tangential $(\mathrm{T})$, normal $(\mathrm{N})$, and combined tangential and normal (TN) EHDs. Most of the curves in the three figures are found to exhibit distinct maxima and minima at specific excitation wavelengths.

For a given set of EHD orientations ( $\mathrm{T}, \mathrm{TN}$, or $\mathrm{N})$, the maximum value of the NRR is found to be constant for all three excitation cases. In particular, for Case $i / T$ ( $i=1,2$, and 3), $\mathrm{NRR}_{\max } \approx 63 \mathrm{~dB}$; for Case $\mathrm{i} / \mathrm{TN}, \mathrm{NRR}_{\max } \approx 68 \mathrm{~dB}$, and for Case $i / \mathrm{N}, \mathrm{NRR}_{\max } \approx 70-71 \mathrm{~dB}$. Thus, for a given excitation case, $\mathrm{NRR}_{\max }$ is larger when normally oriented EHDs are included. These maximum values increase by around $8 \mathrm{~dB}$ from the all-tangential to the all-normal EHD excitations within a specific case. The enhanced response of a normaloriented EHD relative to that of a tangential one was reported previously [27] for the case of a single EHD. This behavior also was found here when multiple EHDs are considered. All maxima of the NRR in Figure 2 occur at the same excitation

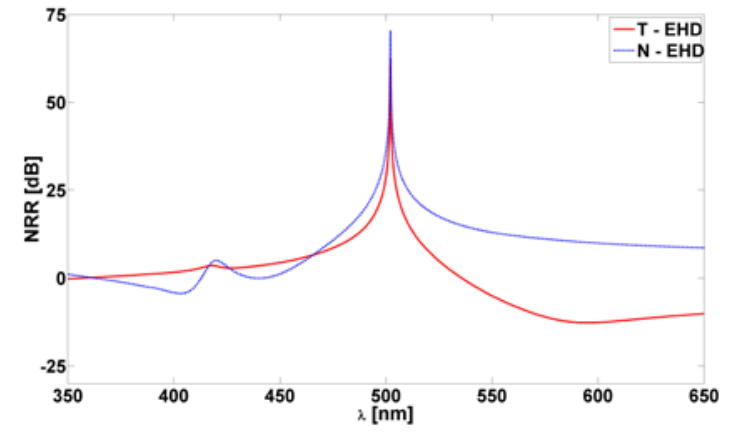

(a) Case $1 / \mathrm{T}$ and $1 / \mathrm{N}$

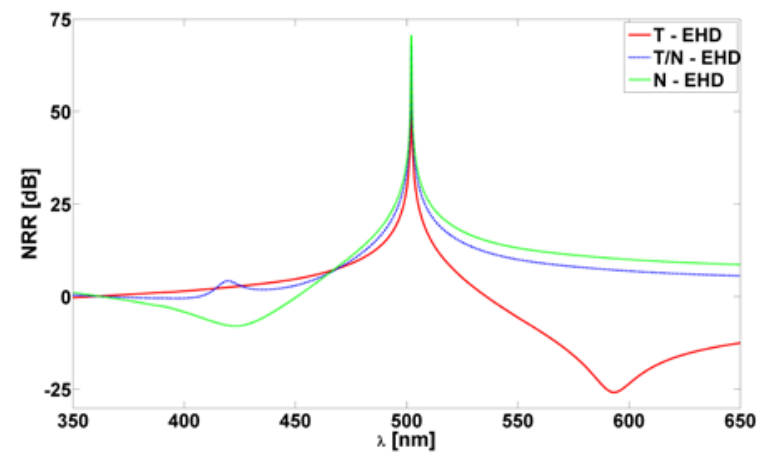

(b) Case $2 / \mathrm{T}, 2 / \mathrm{TN}$, and $2 / \mathrm{N}$

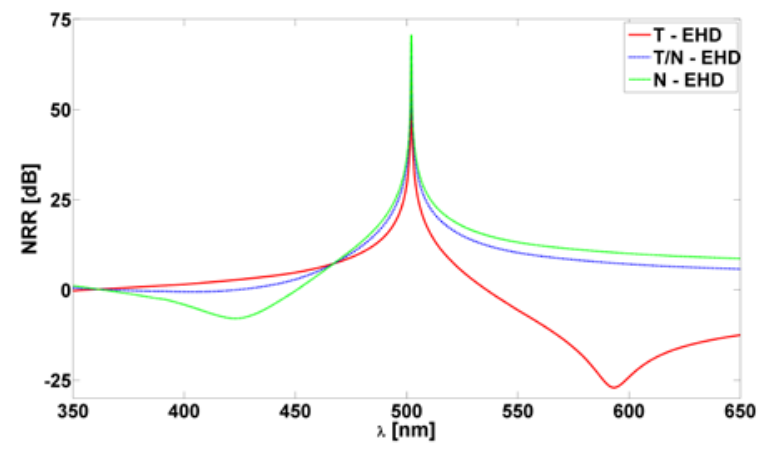

(c) Case $3 / \mathrm{T}, 3 / \mathrm{TN}$, and $3 / \mathrm{N}$

Fig. 2. The NRR (dB) as a function of the free-space wavelength, $\lambda$, for the cases which include one EHD (a), two EHDs (b), and four EHDs (c). In the legends, the symbol $\mathrm{T}(\mathrm{N})$ refers to a configuration with only tangential (normal) EHDs, whereas $\mathrm{TN}$ refers to configurations having both tangential and normal EHDs present.

wavelength: $502.1 \mathrm{~nm}$. These maxima correspond to the super-resonant states $[27,29]$ which are due to a strong excitation of a resonant dipole mode inside the active CNP. This mode is illustrated for the Case $2 / \mathrm{T}$, and $2 / \mathrm{N}$ configurations in Figures 3a) and b), respectively, where the magnitude and direction of their power flow densities are shown in the $x y$-plane.

In regards to the NRR minima observed in Figure 2, we note that a significant dip, corresponding to a large decrease in the radiated power, is in evidence only when all of the EHDs 
were oriented tangential to the CNP. The dip gets substantially shallower when all of the EHDs were oriented normal to the $\mathrm{CNP}$, whereas it is basically non-existing for equal number of tangential and normal EHDs. Moreover, for a given set of EHD orientations ( $T, T N$, or $N$ ), the minima of the NRR curves are not constant across the three excitation cases. In particular, as one goes from Case $1 / \mathrm{T}$ to Case $3 / \mathrm{T}$, the value of $\mathrm{NRR}_{\min }$ goes from $-12.7 \mathrm{~dB}$ to $-27.1 \mathrm{~dB}$ (note that this case was studied in detail in [17]). One the other hand, in going from Case $1 / \mathrm{N}$ to Case $3 / \mathrm{N}$, the value of $\mathrm{NRR}_{\min }$ goes from -4.4 to $-7.8 \mathrm{~dB}$. Thus, for a given excitation case, the magnitude of $\mathrm{NRR}_{\min }$ is largest when all of the EHDs are oriented tangentially; smallest when all of the EHDs are normal-oriented, and non-existing when there is an equal number of tangential- and normal-oriented EHDs.

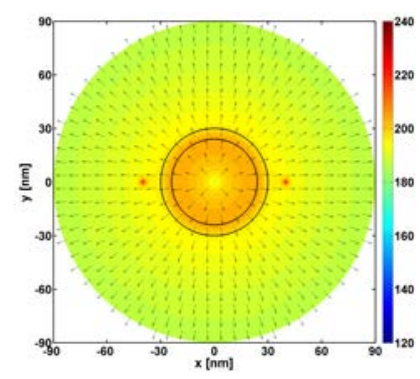

(a)

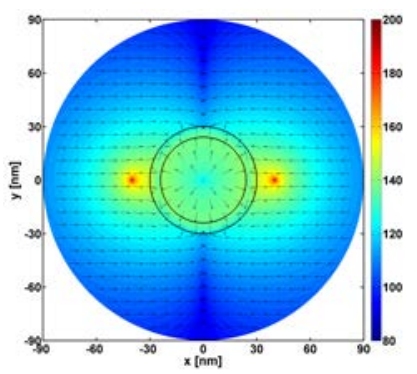

(c)

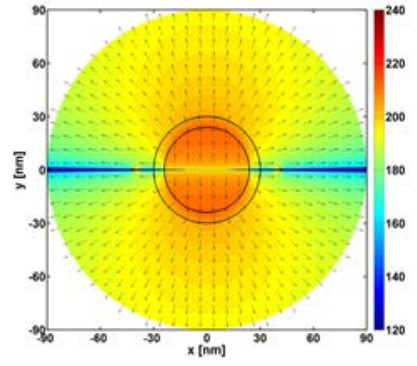

(b)

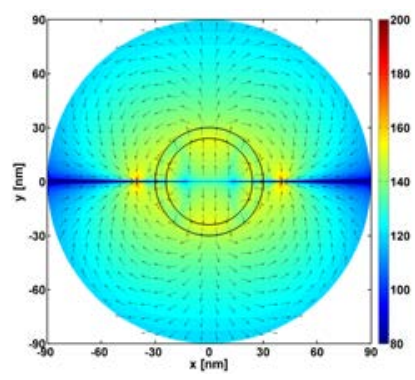

(d)
Fig. 3. Magnitude (color) and direction (arrows) of the power flow density for the Case 2 excitation of the Ag-based CNP corresponding to the peaks, (a) and (b), and dips, (c) and (d), in the NRR in Figure 2(b). Case $2 / \mathrm{T}$ results are shown in (a) and (c). Case $2 / \mathrm{N}$ results are shown in (b) and (d). These power flows are shown in the $x y$-plane in a circular region with a $90 \mathrm{~nm}$ radius. In all cases the EHDs occupy their specified locations in the $x y$-plane at $r_{s}=40 \mathrm{~nm}$. The curves representing the spherical surfaces of the CNP are superimposed in each plot.

It also follows from Figure 2 that the minima in the NRR values are attained at distinct excitation wavelengths. In particular, for Case $i / T, i=1,2$, and 3, they occur at a larger wavelength than the super-resonance wavelength. On the other hand, for

Case $\mathrm{i} / \mathrm{N}, i=1,2$, and 3 , they occur at a wavelength lower than the super-resonance wavelength. These minima in the NRR correspond to the so-called jamming state [27, 29, 35]. This behavior is illustrated in the $x y$-plane for the Case 2/T and Case 2/N configurations given in Figures 3c) and d), respectively.

Table I summarizes the NRR maximum and minimum values and the wavelengths at which they are attained for all three excitation cases and variations of the EHD orientation. It is also useful to note in this Table that the dynamic range (difference between $\mathrm{NRR}_{\max }$ and $\mathrm{NRR}_{\min }$ ) for Case $1 / \mathrm{T}$ and $1 / \mathrm{N}$ is quite the same, whereas the dynamic range for Case $i / \mathrm{T}$, $i=2$ and 3 , is around $10 \mathrm{~dB}$ larger than for the Case $i / \mathrm{N}, i=2$ and 3 , which again is $10 \mathrm{~dB}$ larger than the Case $i / \mathrm{TN}, i=2$ and 3.

TABLE I

MAXIMUM AND Minimum NRR VALUES For CASE 1, 2, AND 3 COHERENT EHD EXCITATIONS.

\begin{tabular}{|c|c|c|c|c|c|c|}
\hline \multirow{2}{*}{ Case } & \multicolumn{3}{|c|}{$\mathrm{NRR}_{\text {max }}[\mathrm{dB}]$} & \multicolumn{3}{|c|}{ Wavelength $\lambda[\mathrm{nm}]$} \\
\hline & $\mathrm{T}$ & $\mathrm{TN}$ & $\mathrm{N}$ & $\mathrm{T}$ & $\mathrm{TN}$ & $\mathrm{N}$ \\
\hline 1 & 62.6 & & 70.4 & & & \\
\hline 2 & 62.9 & 68.1 & 70.7 & All max & occur & $2.1 \mathrm{~nm}$ \\
\hline 3 & 62.9 & 68.4 & 70.7 & & & \\
\hline \multirow{2}{*}{ Case } & \multicolumn{3}{|c|}{$\mathrm{NRR}_{\min }[\mathrm{dB}]$} & \multicolumn{3}{|c|}{ Wavelength $\lambda[\mathrm{nm}]$} \\
\hline & $\mathrm{T}$ & $\mathrm{TN}$ & $\mathrm{N}$ & $\mathrm{T}$ & $\mathrm{TN}$ & $\mathrm{N}$ \\
\hline 1 & -12.7 & & -4.4 & 594.9 & & 403.3 \\
\hline 2 & -25.8 & -0.4 & -7.9 & 593.19 & 396.4 & 422.9 \\
\hline 3 & -27.1 & -0.5 & -7.8 & 593.19 & 403.2 & 422.9 \\
\hline
\end{tabular}

The results in Figure 2 constitute useful far-field signatures that are of potential interest for the determination (sensing) of the overall orientation (in the sense of the tangential and/or normal directions with respect to the CNP) of the molecular environment in which the active CNP is immersed. For instance, if one were to compare two different molecular environments, the one resulting in the larger NRR values will consist predominantly of normally-oriented molecules, while the one resulting in more profound minima in the NRR values will be mostly comprised of tangentially-oriented molecules. Moreover, the dynamic range of the latter will also be larger. These observations can likewise be used to sense the direction of just a single set of molecules. Furthermore, this can be supplemented by careful observations of the wavelength at which the minima in the NRR values are attained relative to the wavelength of the super-resonant state. Recall that the latter does not change with altering orientations of the molecules. The tangential orientation yields minima at a higher wavelength than that of the super-resonant state, whereas the normal orientation provides minima at a lower wavelength. 


\section{B. Phase in-coherent EHDs (molecules)}

In a realistic scenario and in contrast to the previous coherent-radiation results, the phases of the different molecules (EHDs) are very hard to correlate. They will, in general, radiate with different phases. The effect of phase incoherence in the emissions from the individual EHDs on the determination/sensing of their orientation in far-field results can be examined with our model. In this regard, attention is devoted to the Case 2 excitation (EHD A and C). Again, the dipole moment of EHD A is set to: $p_{\mathrm{A}}=5 \mathrm{nA}-\mathrm{m}$, while that of EHD C is set to: $p_{\mathrm{C}}=5 \exp (j \delta) \mathrm{nA}-\mathrm{m}$, i.e., to a different phase, where $\delta=0, \pi / 4, \pi / 2,3 \pi / 4$, and $\pi$ to account for progressively larger degrees of phase in-coherence.

Figure 4 shows the NRR values for Case 2/T (a), Case 2/TN (b), and Case 2/N (c) excitations as a function of the free-space wavelength when the phase of the dipole moment of EHD C is changed. When the two EHDs are both tangential or both normal, the maxima of the NRR (attained at $502.1 \mathrm{~nm}$ ) are largely unaffected (i.e., they decrease only by about $1-2 \mathrm{~dB}$ ) as $\delta$ goes from 0 to $3 \pi / 4$. When the two EHDs radiate completely out of phase, i.e., when $\delta=\pi$, the peak at $502.1 \mathrm{~nm}$ is lost. On the other hand, the magnitude of the minima of the NRR in the case of both tangentially-oriented EHDs decreases with increasing phase in-coherence. In contrast, this is not as nearly pronounced when both EHDs are oriented along the normal directions. Nonetheless, the magnitudes of the minima in the former case are larger in all cases, except for when $\delta=\pi$. In addition, for both of the cases: Case 2/T and 2/N, an additional peak in the NRR occurs at specific lower wavelengths, its peak amplitude increasing with increasing phase in-coherence. Moreover, it is found to be substantially larger when the EHDs are normally-oriented. The second peak in the NRR corresponds to the excitation of a quadrupole mode inside the active CNP. This is confirmed in Figure 5 for the Case $2 / \mathrm{N}$, configuration when $\delta=3 \pi / 4$, which shows the distribution of the magnitude of the $\theta$-component of the electric field, $E_{\theta}$, in a circular region whose radius is $90 \mathrm{~nm}$ in the $x z$-plane. It is further interesting to note that the NRR values for the Case $2 / \mathrm{TN}$ are unaffected by the changing phase of the dipole moment of EHD C.

The larger peak values in the NRR (for all considered degrees of phase in-coherence) and the more pronounced formation of the quadrupole mode for the Case $2 / \mathrm{N}$ configurations, and the larger magnitudes of the NRR minima for all of the Case 2/T configurations but $\delta=\pi$, may constitute a useful far-field signature for sensing the direction of the surrounding in-coherent molecules by the active CNP. Even more interesting, these distinct responses may possibly reveal the degree of in-coherence of a given molecular environment: large dips in the NRR are typical of coherent tangential molecules whereas no profound dips and one or two noticeable peaks (for the presently investigated CNP sizes and EHD locations) indicate the presence of highly in-coherent normally-oriented molecules.

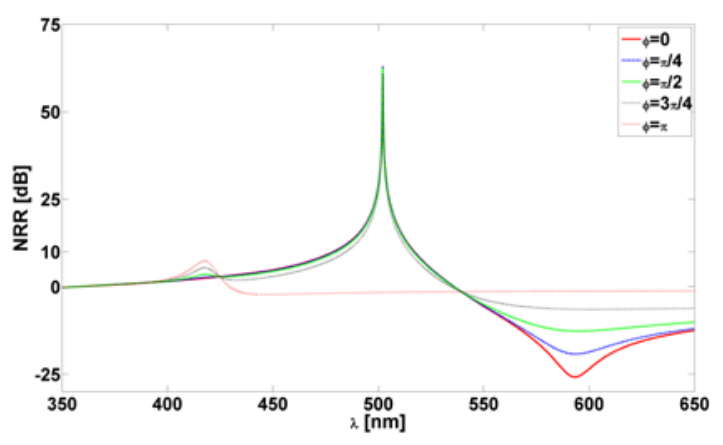

(a) Case $2 / \mathrm{T}$

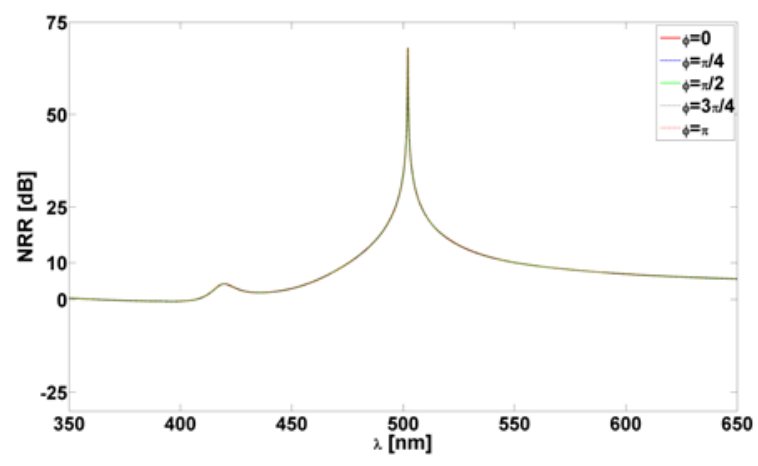

(b) Case $2 / \mathrm{TN}$

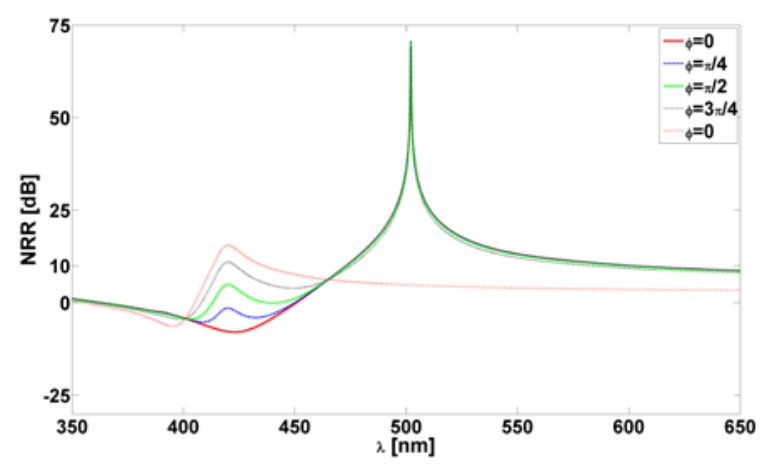

(c) Case $2 / \mathrm{N}$

Fig. 4. The NRR (dB) as a function of the free-space wavelength, $\lambda$, for Case 2/T (a), Case 2/TN (b), and Case 2/N (c) excitations when phase in-coherence between the two EHDs is accounted for. The dipole moment of EHD A is $5 \mathrm{nA}-\mathrm{m}$, while that of EHD C is $5 \exp (j \delta)$ nA-m with $\delta=0, \pi / 4, \pi / 2,3 \pi / 4$ and $\pi$.

\section{CONCLUSIONS}

The potential for using active coated nano-particles to facilitate the determination of molecular orientation was studied analytically. It was demonstrated that the orientation of the molecules produced distinctive signatures in the far field emission data (normalized radiation resistance, NRR). A variety of cases were reported and compared. In particular, 


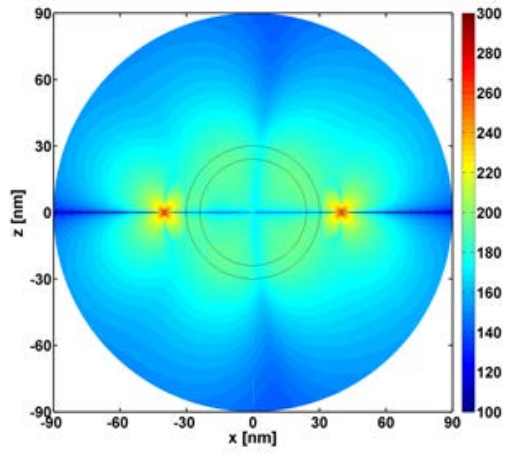

Fig. 5. The $\theta$-component of the electric field, specifically the quantity, $20 \log _{10}\left|E_{\theta} / 1(\mathrm{~V} / \mathrm{m})\right|(\mathrm{dB})$, for the Case $2 / \mathrm{N}$ excitations of the super-resonant active Ag-based CNP associated with additional peak in the NRR in Fig. 4(b) at $420 \mathrm{~nm}$. The results are shown in the $x z$-plane in a circular region having a $90 \mathrm{~nm}$ radius. The two EHDs are located at their respective positions in the $x y$ plane at $r_{s}=40 \mathrm{~nm}$. The dipole moment of EHD A is $5 \mathrm{nA}-\mathrm{m}$, while that of EHD C is $5 \exp (j \delta)$ nA-m with $\delta=3 \pi / 4$. The curves representing the spherical surfaces of the CNP are shown.

it was shown that larger NRR values occur when the dipoles are predominantly normally oriented around the active CNP, whereas more profound minima in the NRR results are due to predominantly tangentially oriented dipoles. Moreover, while the maxima in the NRR values for all dipole orientations occur at the same wavelength, the minima are found at lower (higher) wavelengths for normal (tangential) dipoles relative to the wavelengths of the NRR maxima. The impact of coherent and incoherent phase correlations between the radiating molecules was also described, and similar conclusions apply for the majority of the investigated incoherent phase cases. These investigations also showed that the degree of phase incoherence in a given molecular environment can be revealed by use of active CNPs since large dips in the NRR were typical of coherent tangential molecules, whereas results with no profound dips were attributable to a highly incoherent configuration of normally oriented dipoles. It was thus shown that while the active CNPs can produce extremely large responses in the far-field, they also can act as highly localized, sensitive molecularorientation sensors.

Future work will examine enhancing the sensitivity of active CNPs to sense the orientation of fluorescing molecules by adding additional layers onto a single CNP and by using clusters of the presently investigated CNPs to further tweak the scattering properties of these configurations.

\section{ACKNOWLEDGMENTS}

This work was supported in part by NSF contract number ECCS-1126572.

\section{REFERENCES}

[1] J. R. Lakowicz, ed., 3rd Ed., Principles of Fluorescence Spectroscopy, (Springer, New York, 2006)
[2] L. Novotny, "Single molecule fluorescence in inhomogeneous environments,” Appl. Phys. Lett., vol. 69, pp. 3806-3808, Dec. 1996.

[3] S. Weiss, "Fluorescence spectroscopy of single biomolecules," Science, vol. 283, pp. 1676-1683, Mar. 1999.

[4] Ph. Tamarat, A. Maali, B. Lounis, and M. Orrit, "Ten years of singlemolecule spectroscopy," J. Phys. Chem. A, vol. 104, pp. 1-16, Jan. 2000.

[5] L. Novotny, M. R. Beversluis, K. S. Youngworth, and T. G. Brown, "Longitudinal field modes probed by single molecules," Phys. Rev. Lett., vol. 86, pp. 5251-5254, 2001.

[6] W. E. Moerner, M. Orrit, U. P. Wild and T. Basché, Eds., SingleMolecule Optical Detection, Imaging and Spectroscopy (John Wiley \& Sons, New York, 2008).

[7] T. Ha, T. A. Laurence, D. S. Chemla, and S. Weiss, "Polarization spectroscopy of single fluorescent molecules," J. Phys. Chem. B, vol. 103, pp. 6839-6850, Jun. 1999

[8] B. Sick, B. Hecht, and L. Novotny, "Orientational imaging of single molecules by annular illumination,” Phys. Rev. Lett., vol. 85, pp. 44824485, Nov. 2000.

[9] L. Novotny, M. R. Beversluis, K. S. Youngworth, and T. G. Brown, "Longitudinal field modes probed by single molecules," Phys. Rev. Lett., vol. 86, pp. 5251-5254, Jun. 2001.

[10] M. A. Lieb, J. M. Zavislan, and L. Novotny, "Single-molecule orientations determined by direct emission pattern imaging," J. Opt. Soc. Am. B, vol. 21, pp. 1210-1215, Jun. 2004.

[11] E. M. Purcell, "Spontaneous emission probabilities at radio frequencies," Phys. Rev., vol 69, 681, Jun. 1946.

[12] R. R. Chance, A. Prock, and R. Silbey, "Molecular fluorescence and energy transfer near interfaces,” Adv. Chem. Phys., vol. 37, pp. 1-65, 1978.

[13] P. Anger, P. Bharadwaj, and L. Novotny, "Enhancement and quenching of single-molecule fluorescence,” Phys. Rev. Lett., vol. 96, 113002, Mar. 2006.

[14] S. Kühn, U. Håkanson, L. Rogobete, and V. Sandoghdar, "Enhancement of single-molecule fluorescence using a gold nanoparticle as an optical nanoantenna," Phys. Rev. Lett., vol. 97, 017402, Jul. 2006.

[15] T. H. Taminiau, F. D. Stefani, F. B. Segerink, and N. van Hulst, "Optical antennas direct single-molecule emission," Nature Photon., vol. 2, pp. 234-237, Apr. 2008.

[16] S. Arslanagić, R. W. Ziolkowski, and O. Breinbjerg, "Radiation properties of an electric Hertzian dipole located near-by concentric metamaterial spheres" Radio Sci., vol. 42, RS6S16, doi:10.1029/2007RS003663, Nov. 2007.

[17] S. Arslanagić and R. W. Ziolkowski, "Jamming of quantum emitters by active coated nano-particles,” IEEE J. Sel. Topics Quantum Electron., vol. 19, no. 3, 4800506, May/Jun. 2013.

[18] S. Arslanagić and R. W. Ziolkowski, "Active coated nano-particle excited by an arbitrarily located electric Hertzian dipole - resonance and transparency effects,” J. Opt. A, vol. 12, 024014, Feb. 2010.

[19] P. Bharadwaj, B. Deutsch, and L. Novotny, “Optical antennas,” Adv. Opt. Photon., vol. 1, no. 3, pp. 438-483, Aug. 2009.

[20] L. Novotny and B. Hecht, Principles of Nano-Optics, Cambridge Universtiy Press: New York, 2012.

[21] R. W. Ziolkowski and A. Erentok, "Metamaterial-based efficient electrically small antennas,” IEEE Trans. Antennas Propagat., vol. 54, pp. 2113-2130, Jul. 2006.

[22] A. Erentok and R. W. Ziolkowski, "A hybrid optimization method to analyze metamaterial-based electrically small antennas,” IEEE Trans. Antennas Propag., vol. 55, no. 3, pp. 731-741, Mar. 2007.

[23] R. W. Ziolkowski and A. Erentok, "At and beyond the Chu limit: passive and active broad bandwidth metamaterial-based efficient electrically small antennas," IET Microwaves, Antennas \& Propagation, vol. 1, no.1, pp. 116-128, Feb. 2007.

[24] J. A. Gordon and R. W. Ziolkowski, "The design and simulated performance of a coated nano-particle laser," Opt. Express, vol. 15, pp. 2622-2653, Mar. 2007.

[25] J. A. Gordon, and R. W. Ziolkowski, "Investigting functionalized active coated nano-particles for use in nano-sensing applications," Opt. Express, vol. 15, pp. 12562-12582, Oct. 2007. 
[26] J. A. Gordon, and R. W. Ziolkowski, “Optical CNP metamaterials,” Opt. Express, vol. 16, pp. 6692-6716, Apr. 2008.

[27] S. Arslanagic and R. W. Ziolkowski, "Active coated nano-particles: Impact of plasmonic material choice,” Appl. Phys. A, vol. 103, pp. 795798, Jun. 2011.

[28] S. D. Campbell and R. W. Ziolkowski, "Impact of strong localization of the incident power density on the nano-amplifier characteristics of active coated nano-particles," Opt. Commun., vol. 285, pp. 3341-3352, Jul. 2012.

[29] R. W. Ziolkowski, S. Arslanagić, and J. Geng, "Where high-frequency engineering advances optics: Active nanoparticles as nanoantennas," in Optical Nanoantennas, Eds. A. Alú and M. Agio, Cambridge Universiy Press: London, 2013.

[30] H. W. Chew, P. J. McNulty, and M. Kerker, "Model for a Raman and fluoroscent scattering by molecules embedded in small particles," Phys. Rev. A., vol. 13, pp. 396-404, 1976.

[31] N. G. Alexopoulos, and N. K. Uzungolu, "Electromagnetic scattering from active objects: invisible scatterers,”. Appl. Opt., vol. 17, pp. 235232, 1978.

[32] A. Alú, and N. Engheta, "Plasmonic and metamaterial cloaking: physical mechanisms and potentials,” J. Opt. A:Pure Appl. Opt., vol. 10, 093002, Jul. 2008.

[33] N. J. Halas, "Plasmonics: An emerging field fostered by Nano Letters," Nano. Lett., vol. 10, pp.3816-3822, Sep. 2010.

[34] I. Choi and Y. Choi, "Plasmonic nanosensors: review and prospect," IEEE J. Selected Top. Quantum Electron., vol. 18, Nr. 3, pp. 1110-1121, May/June, 2012.

[35] S. Arslanagić, S.D. Campbell and R. W. Ziolkowski, "Passive and Active Nano-antenna Systems," in Singular and Chiral Nanoplasmonics, S. Boriskina and N. Zheludev, Eds., Pan Stanford Publishing: Singapore, 2014. 